\title{
Successful Cessation of Transmitting Healthcare-Associated Infections due to Burkholderia CEPACIA COMPlEX in A NeONATAl Intensive Care Unit in a Japanese Children's Hospital
}

\author{
K. Kuzumoto ${ }^{1}$, N. Kubota ${ }^{1}$, K. Ishii2, ${ }^{2}$, K. Yumoto ${ }^{1}$, Y. Ogiso ${ }^{1,3}$, T. Nakamura ${ }^{4}$, T. Katsuyama ${ }^{1,3}$, \\ K. Oana ${ }^{5,6}$, Y. Kawakami 5, 6
${ }^{1}$ Department of Laboratory Medicine, ${ }^{2}$ Department of Nursing, ${ }^{3}$ Department of Infection Control, ${ }^{4}$ Division of Neonatology, Nagano Children's Hospital, Azumino, Japan,
${ }^{5}$ Division of Infection Control and Microbiological Regulation, Department of Health and Medical Sciences, Shinshu University Graduate School of Medicine,
${ }^{6}$ Division of Clinical Microbiology, Department of Biomedical Laboratory Sciences, School of Health Sciences, Shinshu University School of Medicine, Matsumoto, Japan

\begin{abstract}
Background: Burkholderia cepacia strains have been known to possess the capability to cause serious infections especially in neonatal intensive care units (NICUs), and their multi-drug resistances become a severe threat in hospital settings. The aim of this investigation was to evaluate the $B$. cepacia complex infections in the NICU in Nagano Children's Hospital, Azumino 399-8288, Japan, and to report the intervention leading to the successful cessation of the outbreak.

Methodology: The incidence of isolation and antimicrobial susceptibilities of nosocomial Burkholderia cepacia complex strains during a four-year period were retrospectively examined by clinical microbiological records, and by pulsed-field gel electrophoresis analyses along with the bacteriological verification of disinfectant device itself and procedures for its maintenance routinely used in the NICU.

Results: During the period surveyed between 2007 and 2009 , only an isolate per respective year of B. cepacia complex was recovered from each neonate in the NICU. However, in 2010, the successive 6 B. cepacia complex isolates were recovered from different hospitalized neonates. Among them, an isolate was originated from peripheral blood of a neonate, apparently giving rise to systemic infection. In addition, the hospitalized neonate with bacteremia due to $B$. cepacia complex also exhibited positive cultures from repeated catheterized urine samples together with tracheal aspirate secretions. However other 5 isolates were considered as the transients or contaminants having little to do with infections. Moreover, the 5 isolates between July and October in 2010 revealed completely the same electrophoresis patterns by means of pulsedfield gel electrophoresis analyses, strongly indicating that they were infected through the same medical practices, or by transmission of the same contaminant. Conclusions: A small outbreak due to B. cepacia comlex was brought about in the NICU in 2010, which appeared to be associated with the same genomovar of $B$. cepacia complex. The source or the rout of infec-
\end{abstract}

tion was unknown in spite of the repeated epidemiological investigation. It is noteworthy that no outbreak due to $B$. cepacia complex was noted in the NICU after extensive surveillance intervention.

Key words: nosocomial infection, health-care-associated infection, NICU, Burkholderia cepacia, surveillance, disinfectant contamination

\section{INTRODUCTION.}

Burkholderia cepacia complex strains are consisted of closely related species sharing a $16 \mathrm{~S}$ rRNA gene sequence with high similarity $(>97.5 \%)$ comprising of at least 17 validly named species and 15 genospecies [1-3], and have been increasingly recognized as important healthcare-associated infectious agents in hospitalized and immunocompromised patients [4-6]. Strains of $B$. cepacia complex are the common inhabitants found in moist hospital environment $[7,8]$, reflecting its intrinsic aptitude to survive and grow in humid environments with minimal nutrition. Indeed, hospital outbreaks have usually been due to common contaminated sources such as disinfectant products, intravenous solutions, nebulizer solutions, or medical devices [9-12].

During the routine clinical microbiological examinations, we encountered successive 6 isolations of $B$. cepacia complex between April and October in 2010 from different newborn babies in the neonatal intensive care unit (NICU) in Nagano Children's Hospital with 200 beds, Azumino 399-8288, Japan. Emergence of this event in 2010 led us to review the hospital's microbiological records for $B$. cepacia complex colonization or infection, during the last 4 years, that is, from April in 2007, through October in 2010, until March in 2011.

We conducted an epidemiological investigation of B. cepacia complex colonization or infection in a neonatal intensive care unit (NICU) of newborn-baby ward. The newborn-baby ward is constituted of both an NICU with 21 beds and a growing care unit (GCU) with 18 beds. 
Colonization was defined by the isolation of $B$. cepacia complex from clinical specimens, in cases that criteria for infection were not satisfied notwithstanding its rate of isolation. Respiratory infections of neonates were defined by radiological and/or clinical criteria, including the isolation of $B$. cepacia complex from tracheal aspirates $\left(10^{5}\right.$ colony-forming units $[\mathrm{CFU}] / \mathrm{ml}$ or more). Urinary tract infections were defined by observing $10^{4}$ colony-forming units $[\mathrm{CFU}] / \mathrm{ml}$ or more of bacterial cells. Systemic bloodstream infections of the neonates were defined by one or more positive blood cultures. Colonization or infection was considered to be hospital-acquired when it occurred at least 48 hours after their births in our hospital.

\section{Methods}

\section{Clinical Epidemiology Analyses}

The numbers of emergence and the dates of isolation of $B$. cepacia complex from the neonates in the NICU were inspected from April in 2007, through December in 2010, until March in 2011, whether or not the isolates were considered as causative agents, residents or contaminants. Biochemical characterization and identification together with the antimicrobial susceptibilities of the isolates were carried out with the MicroScan WalkAway 40 System (Siemens Healthcare Diagnostics, Tokyo, Japan) using the Neg Combo 3.12J panels (Siemens Healthcare Diagnostics, Tokyo, Japan) in the system according to the manufacture's instructions. Commercially available kit systems, ID-Test NF18 panels (Nissui Pharmaceuticals Co., Ltd., Tokyo, Japan.) were used, when necessary, for confirmation of biochemical properties. We then compared the isolation rate during the epidemic period (between July and October in 2010) with that ahead of the outbreak (between April in 2007 and March in 2010).

Between April and October in 2010, the different 6 neonates successively yielded positive cultures for $B$. cepacia complex strains in routine clinical microbiology laboratory examinations. Among the 6 neonates, recent 5 neonates with overlapping periods of hospitalization was molecular epidemiologically analyzed by means of pulsed-field gel electrophoresis to investigate their genetic relatedness [13, 14]. Isolates were grown in $2 \mathrm{ml}$ of Brain-Heart-Infusion (Nissui Pharmaceuticals Co., Ltd., Tokyo, Japan.) broth media at $35^{\circ} \mathrm{C}$ for 18 hours, and the DNAs were prepared from the bacterial pellets as previously described [15]. The respective DNA was digested with SpeI (5 -ACTAGT3 ) restriction enzyme (Takara Bio Inc., Shiga, Japan.), and analyzed according to the manufacturer's instructions. Two unrelated isolates that were recovered from the infant inpatients in other different wards were included in the pulsed-field gel electrophoresis analyses for comparison.

\section{Microbiological Review of Routine Procedures FOR DISINFECTION}

Procedures for the use, maintenance, and disinfection of the respiratory therapy devices in the NICU were observed and reviewed. The three experts consisting of two infection control doctors (ICDs) and an infection control nurse (ICN) from outside the ward conducted the procedural audit. It focused on all respiratory manipulations, including secretion aspiration, intubation, and mechanical ventilation. Special attention was paid to the handling of disinfectant-equipments.

During the epidemic period of October days in 2010, we collected all of the disinfectant-containing bottles from each hospital personnel and from environmental sites in the NICU. A total of 104 disinfectant fluids in bottles consisting of 37 ethyl-alcoholic solution with glycerin, 13 ethyl-alcoholic chlorhexidine-digluconate, 13 ethyl-alcoholic benzalkoniumchloride, together with 34 hand-washing soap solutions and 7 lotion fluids for hand care, were properly diluted in a ten-fold fashion, and then plated duplicate (100 $\mu$ l, respectively) on Trypticase-Soy agar plates (Eiken Chemical Co., Ltd., Tokyo, Japan.), or $1 \mathrm{ml}$ of respective undiluted disinfectant, hand-washing soap, or lotion fluid for hand care was put into $15 \mathrm{ml}$ of Brain-Heart-Infusion (Nissui Pharmaceuticals Co., Ltd., Tokyo, Japan.) broth media and then incubated at $35^{\circ} \mathrm{C}$ for 72 hours. Cotton-swab samples from surfaces of respiratory therapy devices, infant incubators, and keyboards of personal computers in the NICU were spread onto Sheep Blood Agar (Nippon Becton Dickinson Co., Ltd., Tokyo, Japan.) plates and incubated at $35^{\circ} \mathrm{C}$ for 72 hours.

In addition, we tried to verify the hand-washing procedures by the ICN in our hospital, concerning 9 link nurses from all of the divisions consisting of 7 clinical wards including NICU of newborn-baby ward, 1 operation room, and 1 outpatient clinic department, and together with 50 full-time medical doctors in the Nagano Children's Hospital, by means of palm-stamp methods using SCDLP agar (Palm-Stamp-Check) plates (Nikken Bio Medical Laboratory Inc., Kyoto, Japan). A total of 57 medical personnel were microbiologically surveyed by the ICN for the exaltation of improving routine healthcare infection control practices. The number of bacteria was counted by a palm stamp check agar plate method for general bacteria before and after hand-washing.

\section{RESULTS}

\section{Analytical Epidemiology of B. CEPACIA COMPLEX ISOLATIONS}

As shown in Figure 1, the 9 neonates (from Patient-1 to Patient-9) in the NICU grew B. cepacia complex; of these, as for a total of 3 neonates (i.e., Patient-1, -2, and -3 ) every 1 isolate a year was recovered from nasal secretions or tracheal aspirates as colonization or contamination, between April in 2007 and March in 2010. The remaining 6 neonates yielded $B$. cepacia complex between April in 2010 and October in 2010, considered being a small outbreak. The breakdown of the outbreak occurred was as follows; a premature neonatal Patient-4 with congenital thorax disease on April 4 from nasal secretion and then on April 27 from nasal secretion and tracheal aspirate, a chromosomal abnormality neonatal Patient -5 with congenital heart disease on July 13 from tracheal aspirate, and a premature neonatal Patient-6 with extremely low birth weight on 
September 14 from tracheal aspirate, and successively from both peripheral blood and catheterized urine on September 21, and a premature neonatal Patient-7 with respiratory distress on September 14 from tracheal aspirate, a pyruvate dehydrogenase deficient neonatal Patient-8 with respiratory ventilation on September 28 from tracheal aspirate, and then an extremely low-birth-weight infant Patient-9 with trisomy-18 syndrome and with congenital diseases from tracheal and nasal secretions. The hospitalization of the latter 5 neonates (P-5, -6, -7, -8, and -9) yielding $B$. cepacia complex wholly overlapped.

\section{Microbiological Analyses of EnVironments And Procedures RevieW}

In the examination of the 5 clinical isolates from different 5 neonates (Patient-5, -6, -7, -8, and -9), the Neg Combo 3.12J panels in the MicroScan WalkAway 40 plus system represented almost entirely the same profile number (data not shown) and almost exactly the same antibiograms with one another as shown in Table 1, demonstrating that the most active agent was meropenem (MIC-values, less than $1 \mu \mathrm{g} / \mathrm{ml}$ ) followed by minocycline and sulfisoxazol/trimethoprim (both MIC-values, less than $2 \mu \mathrm{g} / \mathrm{ml}$ ), and new quinolone drugs of levofloxacin and ciplofloxacin (both MICvalues, less than 0.5 or $1 \mu \mathrm{g} / \mathrm{ml})$. However, the 2 isolates (Patient-A and Patient-B) derived from the wards unrelated to the NICU for comparison revealed the different antibiograms, as shown in Table 1, that is, both 2 displayed resistances to new quinolone drugs of levofloxacin and ciplofloxacin (both MIC-values, 2 $\mu \mathrm{g} / \mathrm{ml}$ or more), one with resistance to ceftazidime (MIC-values, more than $16 \mu \mathrm{g} / \mathrm{ml}$ ), and the another with resistance to minocycline (MIC-values, $4 \mu \mathrm{g} / \mathrm{ml}$ ), in distinctive contrast to the epidemic isolates from the neonatal Patient-5, $-6 \mathrm{a},-6 \mathrm{~b},-7,-8$, and -9 .

Moreover, as shown in Figure 2, the pulsed-field gel electrophoresis analyses of the 6 isolates (each 1 isolate from Patient-5, $-7,-8$, and -9 , and 2 isolates from the different specimens of Patient- 6 designated as $\mathrm{Pa}$ tient-6a and -6b) yielded indistinguishable electrophoresis patterns, with the two different patterns from diverse isolates in other wards for comparison.

No B. cepacia complex isolate was recovered from all of the medical devices, equipments or the keyboards of personal computers. The later keyboards were overlapped with the very dedicated own covers being changed three-times a day for the disinfected ones treated with ethyl-alcoholic disinfectants.

Repeated isolation trials failed to detect B. cepacia complex from any of the 104 disinfectant fluids in bottle devices that the personnel in the NICU always wearing around their own waists, and that already arranged in the appropriate sites in the ward.

Microbiological verification of hand-washing procedures by hospital personnel in the NICU was performed with the palm-stamp methods. It should be noticeable that no growth of colonies indicative of $B$. cepacia complex occurred and that the obvious decrease of general bacterial cell counts was manifested almost all the personnel investigated, after their usual hand-washing procedures using any of the hand-wash-
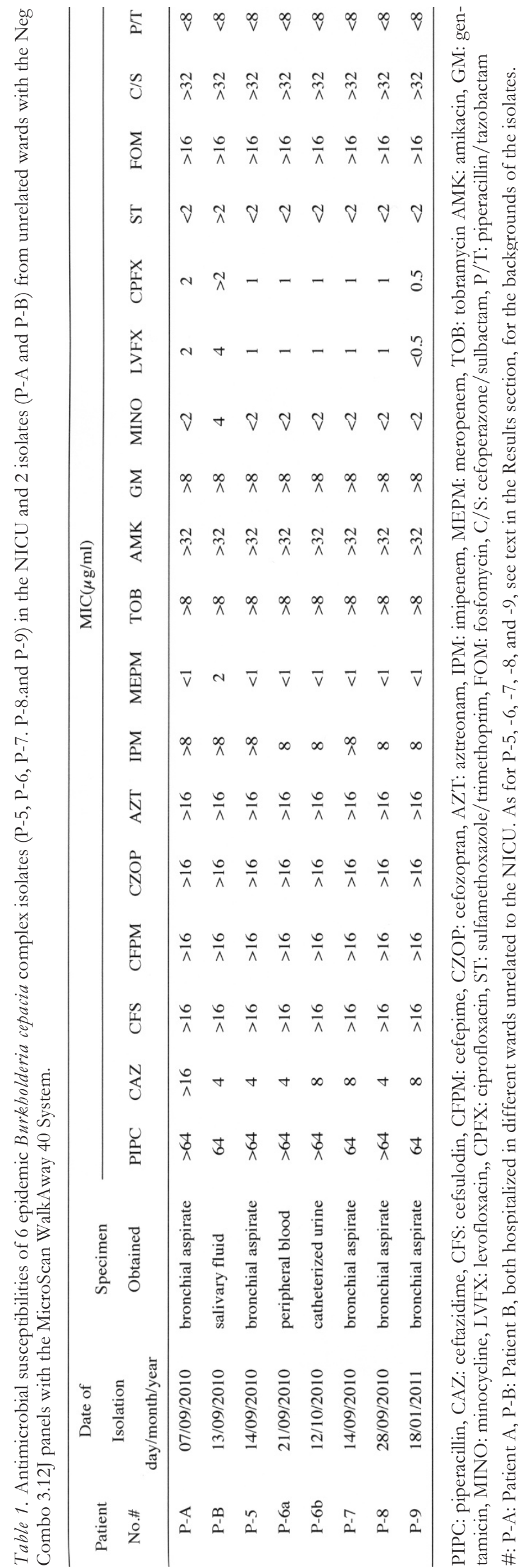
ing fluids, except for 2 link nurses and 7 full-time medical doctors. That is, 9 out of 57 hospital personnel surveyed had to attend the lectures on hand-washings and on hygiene, and then they were to be further rechecked the processing of hand-washings by the ICN.

\section{Discussion}

Burkholderia cepacia complex strains are problematic pathogens in the hospital settings because of their high resistances to antimicrobials, of making infections difficult to treat, and of their innate potentials to survive or inhabit in every moist hospital environment. Moreover, they are difficult to identify and additional new species have now been found to constitute isolates originally identified as $B$. cepacia complex based on their phenotypic properties [1-3]. Indeed, the taxonomy of the $B$. cepacia complex has advanced considerably along with the molecular biological development for their accurate identification to the genospecies level [3]).

As shown in Figure 1, during the period between April in 2007 and March in 2010, the 3 B. cepacia complex isolates from 3 different neonates (Patients-1, -2 , and -3 ) were recovered in the NICU from the clinical specimens; Patient- 1 from nasal secretion in 2007 and Patients- 2 and -3 from tracheal aspirates both at a density of less than $10^{3} \mathrm{CFU} / \mathrm{mL}$ in 2008 and 2009, respectively) with no clinical sigh of infections, and therefore every $B$. cepacia complex isolate was considered as a transient contaminant. In the subsequent analyses during the period between April and October in 2010 , the 5 (Patients-4, -5, -7, -8 and -9 ) out of the 6 neonates yielding $B$. cepacia complex isolates were born in our hospital and successively hospitalized in the NICU during the overlapping periods. The residual 1 neonatal Patient- 8 was promptly hospitalized in the NICU in our hospital soon after being transported from different hospital.

That is to say, all of the 9 acquisitions of B. cepacia complex were diagnosed as hospital-acquired. In particular, all of the 5 neonates (P-5, -6, -7, -8, and -9) yielding B. cepacia complex isolates between September and October in 2010 had been hospitalized in the NICU during the absolutely overlapping periods, raising the possibility of neonate-to-medical personnel or -medical equipments-to-neonate transmission.

Therefore we discriminate the 5 epidemic isolates by means of pulsed-field gel electrophoresis analyses, and found that all of the 5 isolates exhibited completely identical electrophoresis patterns, as clearly demonstrated in Figure 2, indicating that they should be the causative agents of healthcare-associated infections or transmissions during the overlapping periods due to the same contaminant origin in the NICU. $B$. cepacia complex strains are easily transmitted from patient to patient through close contact or through environmental acquisition $[16,17]$. In order to prevent the transmission, important aspects concern the disinfectant equipments, because $B$. cepacia complex strains may contaminate the devices and may survive by resisting against the disinfectant fluids [18]. Therefore we made sure by microbiologically checking whether the disinfectant fluids were contaminated with $B$. cepacia complex or not. The disinfectant devices are not only placed in predefined positional places but also carried all the time by respective personnel in the NICU with their waists and are always utilized in cases disinfection was needed. We microbiologically exam-

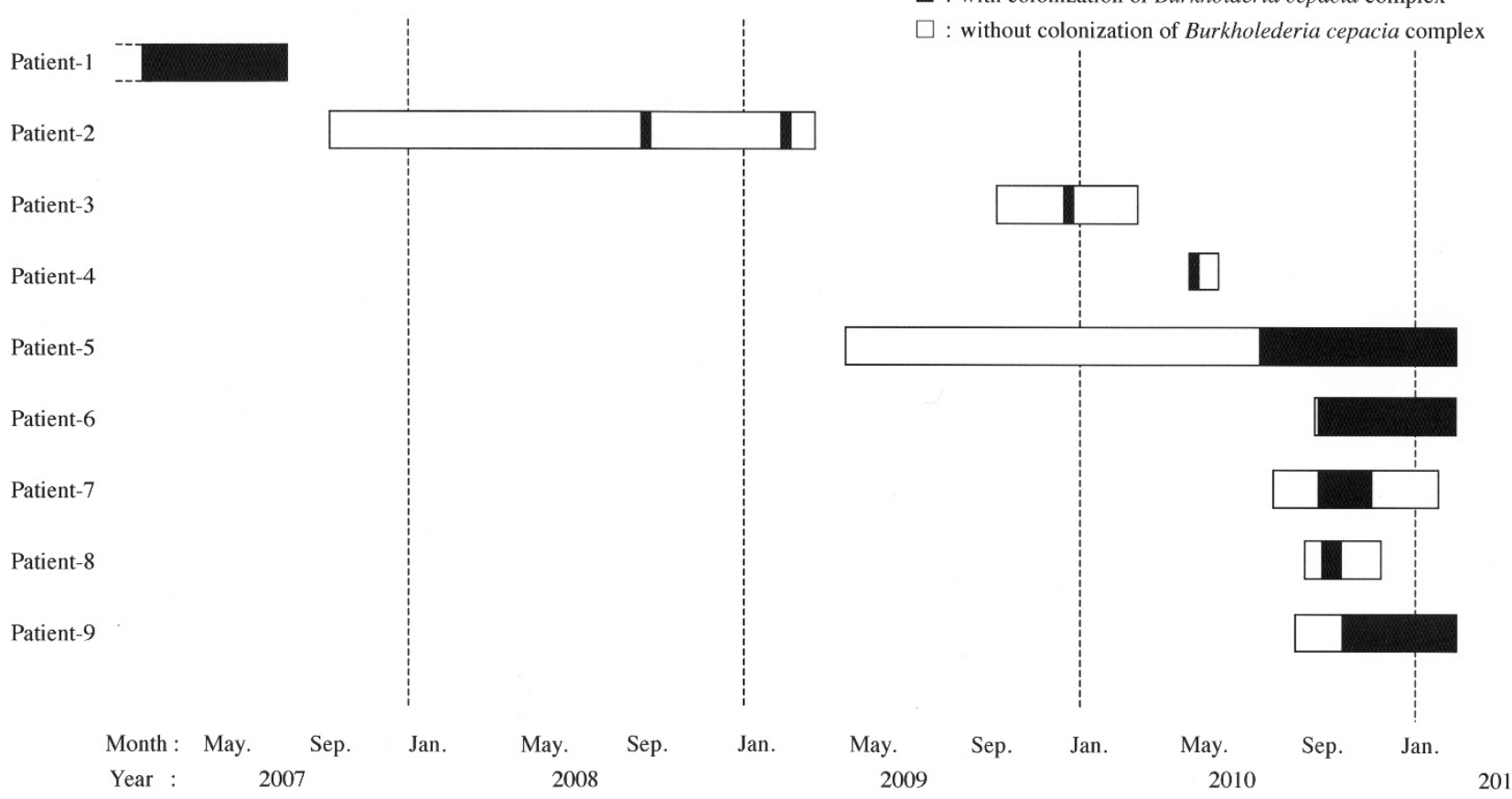

Fig. 1. Time course of Burkholderia cepacia complex outbreak in the neonatal intensive care unit (NICU) from April in 2007 through October in 2010 to March in 2011. Each bar represents the hospitalization period of each inpatient neonate. See Table 1 , for the origins, and see text in the Results section, for the backgrounds of the isolates. 


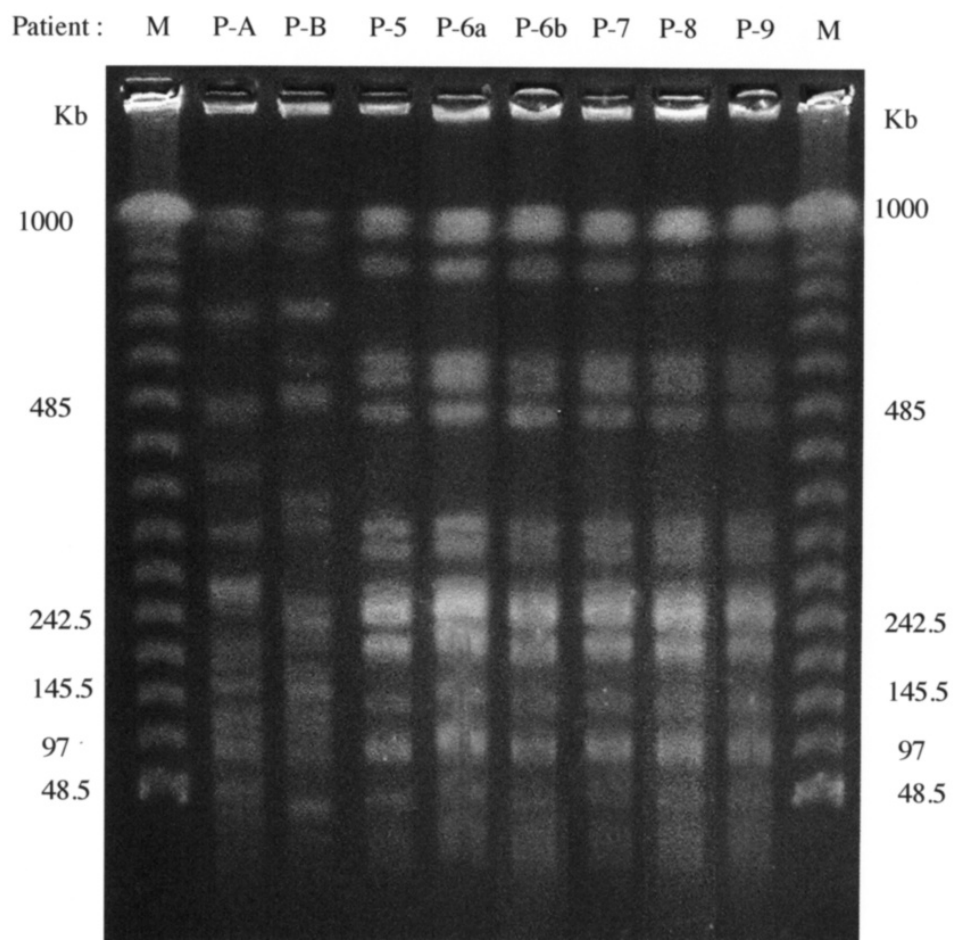

Lanes both sides M: lambda DNA ladder as molecular size markers (in Kb). P-A: Patient A, P-B: Patient B, both from different wards unrelated to the NICU. P-5: Patient-5, P-6a and P-6b: Patient-6, P-7: Patient-7, P-8: Patient-8, and P-9: Patient-9, respectively. See Table 1, for the origins, and see text in the Results section, for the backgrounds of the isolates.

Fig. 2. Profiles of the genomic DNAs of 6 epidemic Burkholderia cepacia complex after digestion with SpeI (5 -ACTAGT-3) restricted enzyme in the neonatal intensive care unit, together with 2 Burkholderia cepacia complex isolates in different wards, for comparison.

ined all of the 104 fluid samples from 104 different disinfectant devices in the NICU, however, no isolation of $B$. cepacia complex was observed even after the repeated investigation trials. It should be challenging and difficult to find out the origin, source or the rout of infection with $B$. cepacia complex.

Although the examinations for general bacterial counts before and after the hand-washing was indeed useless to clarify the origin or the rout of infections due to $B$. cepacia complex, it should be noted that the hand-washing is fundamentally basic and imperative in controlling healthcare-associated infections or transmissions. Moreover, we should anticipate that full-time medical doctors as well as link nurses would play an important role in their own clinical area to facilitate liaison with the colleagues and other surrounding medical personnel in routine infection control practices $[19,20]$.

No environmental source or no obvious mechanism of transmission of $B$. cepacia complex was found in the NICU of newborn ward. However, since October in 2010 when these microbiological examinations had been carried out in the NICU, what is noteworthy is no new cases of colonization or infection with $B$. cepacia complex have occurred for the last 5 months, despite the presence of neonatal inpatients colonized with $B$. cepacia complex on the ward. This led us to consider that the reinforcement of, or stepping into hygienic measures should give the deterrent effect against the spread of the infections.

This study illustrates a value of careful epidemiologic investigation in controlling the colonization or infections due to $B$. cepacia complex. Complete elimination of the transmission among the neonates was achieved only after the intervention of investigating microbiologically the environmental sites in the NICU, particularly the 104 disinfectant devices that every medical personnel in the NICU always bring in each hip pouch around each waist. These active microbiological interventions might actually lead to the cessation of the outbreak, despite the fact that the source or the rout of infection due to B. cepacia complex in the NICU was not found.

Conflicts of interest: The authors have declared that no conflict of interest exists.

\section{REFERENCES}

1. Coenye T, Vandamme P, Govan JR, LiPuma JJ. Taxonomy and identification of the Burkholderia cepacia complex. J Clin Microbiol. 2001; 39: 3427-3436.

2. Vandamme P, Holmes B, Vancanneyt M, Coenye T, Hoste B, Coopman R, Revets H, Lauwers S, Gillis M, Kersters K, Govan JR. Occurrence of multiple genomovars of Burkholderia cepacia in cystic fibrosis patients and proposal of Burkholderia multivorans sp. nov. Int J Syst Bacteriol. 1997; 47: 1188-1200.

3. Vandamme P, Dawyndt P. Classification and identification of the Burkholderia cepacia complex: present and future. Syst Appl Microbiol. 2011; 34: 87-95.

4. Jones AM, Dodd ME, Webb AK. Burkholderia cepacia: current clinical issues, environmental controversies and ethical dilemmas. Eur Respir J. 2010; 17: 295-301.

5. Webb AK, Egan J. Should patients infected with Burkbolderia cepacia undergo lung transplantation. Thorax 1997; 52: 671- 673.

6. Snell GI, de Hoyos A, Krajden M, Winton T, Maurer JR. Pseudomonas cepacia in lung transplant recipients with cystic fibrosis. Chest 1993; 103: 466-471. 
7. Thomassen MJ, Demko CA, Klinger JD, Stern RC. Pseudomonas cepacia colonisation among patients with cystic fibrosis. A new opportunist. Am Rev Resp Dis. 1985; 131: 791-796.

8. LiPuma JJ, Dansen SE, Nielson DW, Stern RC, Stull TL. Person-to-person transmission of Pseudomonas cepacia between patients with CF. Lancet. 1990; 336: 1094 1096.

9. Loukil C, Saizou C, Doit C, Bidet P, Mariani-Kurkdjian P, Aujard Y, Beaufils F, Bingen E. Epidemiological investigation of Burkholderia cepacia acquisition in two pediatric intensive care unit. Infect Control Hosp Epidemiol. 2003; 24: 707-710.

10. Dizbay M, Tunccan OG, Sezer BE, Aktas F, Arman D. Nosocomial Burkholderia cepacia infections in a Turkish university hospital: a five-year surveillance. J Infect Dev Ctries. 2009; 3: 273-277.

11. Jones AM, Dodd ME, Webb AK. Burkholderia cepacia as a cause of ecthyma gangrenosum-like lesion. Infection 2001; 36: 271-273.

12. Mahenthiralingam E, Urban TA, Goldberg JB. The multifarious, multireplicon Burkholderia cepacia complex. Nat Rev Microbiol. 2005; 3: 144-156.

13. Oana K, Kawakami Y, Hayashi T, Ohnishi M. Simple broad-spectrum protocol using labiase for bacterial cell lysis to prepare genomic DNA for pulsed-field gel electrophoresis analysis. Microbiol Immunol. 2009; 53: 45-48.

14. Oana K, Kawakami Y, Ohnishi M, Ishikawa M, Hirota M, Tozuka M, Atarashi K, Baba K, Fujiki K, Okazaki M, Honda T, Hayashi T. Molecular and epidemiological study of the first outbreak of vanB type vancomycin-resistant Enterococcus faecalis in Japan. Jpn J Infect Dis. 2001; 54: 17-22.

15. Coenye T, Spilker T, Martin A, LiPuma JJ. Comparative assessment of genotyping methods for epidemiologic study of Burkholderia cepacia genomovar III. J Clin Microbiol. 2001; 40: 3300-3307.
16. Hutchinson GR, Parker S, Pryor JA, Duncan-Skingle F, Hoffman PN, Hodson ME, Kaufmann ME, Pitt TL. Home-use nebulizers: a potential primary source of Burkbolderia cepacia and other colistin-resistant, gram-negative bacteria in patients with cystic fibrosis. J Clin Microbiol. 1996; 34: 584-587.

17. Saiman L, Siegel J. Infection control in cystic fibrosis. Clin Microbiol Rev. 2004; 17: 57-71.

18. Hakuno H, Yamamoto M, Oie S, Kamiya A. Microbial contamination of disinfectants used for intermittent self-catheterization. Jpn J Infect Dis. 2010; 63: 277-279.

19. Dawson SJ. The role of the infection control link nurse. J Hosp Infect. 2003; 54: 251-257.

20. Teare EL, Peacock A. The development of an infection control link-nurse programme in a district general hospital. J Hosp Infect. 1996; 34: 267-278.

Received: March 23, 2011 / Accepted: July 7, 2011

Address correspondence:

Yoshiyuki Kawakami, Ph.D., Professor

Division of Infection Control and

Microbiological Regulations,

Department of Health and Medical Sciences,

Shinshu University Graduate School of Medicine,

Matsumoto, 390-8621, Japan.

Phone: +81-263-37-2381

Fax: +81-263-37-2370

E-mail: yk23724@shinshu-u.ac.jp 